\title{
Angiotensin-Converting Enzyme Inhibitors (ACEIs) and Angiotensin-Receptor Blockers (ARBs) in Patients at High Risk of Cardiovascular Events: A Meta-Analysis of 10 Randomised Placebo-Controlled Trials
}

\author{
Hean Teik Ong, ${ }^{1}$ Loke Meng Ong, ${ }^{2}$ and Jacqueline Judith $\mathrm{Ho}^{3}$ \\ ${ }^{1}$ Consultant Cardiologist, HT Ong Heart Clinic, 251 C Burma Road, Penang 10350, Malaysia \\ ${ }^{2}$ Consultant Nephrologist and Head, Department of Medicine and Clinical Research Centre, Penang Hospital, Penang 10990, Malaysia \\ ${ }^{3}$ Clinical Epidemiologist, Professor and Head of Paediatrics, Penang Medical College, Penang 10450, Malaysia
}

Correspondence should be addressed to Hean Teik Ong; ongheanteik@gmail.com

Received 11 June 2013; Accepted 2 September 2013

Academic Editors: M. Abdelnoor, A. Bobik, J. Morais, A. Politi, and A. Szekely

Copyright (C) 2013 Hean Teik Ong et al. This is an open access article distributed under the Creative Commons Attribution License, which permits unrestricted use, distribution, and reproduction in any medium, provided the original work is properly cited.

\begin{abstract}
Context. Whether angiotensin converting-enzyme inhibitors (ACEI) and angiotensin-receptor blockers (ARB) are useful in high risk patients without heart failure is unclear. We perform a meta-analysis of prospective randomized placebo-controlled ACEI or ARB trials studying patients with a combination of risk factors to assess treatment impact on all cause mortality, cardiovascular mortality, nonfatal myocardial infarction (MI) and stroke. Method. A PubMed search was made for placebo-controlled trials recruiting at least 1,200 high risk patients randomized to either ACEI or ARB, with follow-up of at least 2 years. Meta-analysis was performed using the RevMan 5 program and Mantel-Haenszel analysis was done with a fixed effects model. Results. Ten trials recruiting 77,633 patients were reviewed. All cause mortality was significantly reduced by ACEI (RR $0.89 ; P=0.0008$ ), but not by ARB treatment (RR 1.00; $P=0.89$ ). Cardiovascular mortality and nonfatal MI were also reduced in the ACEI trials but not with ARB therapy. Stroke was significantly reduced in the ACEI trials (RR $0.75 ; P<0.00001)$ and more modestly reduced in the ARB trials (RR 0.90; $P=0.01$ ). Conclusion. ACEI treatment reduced stroke, nonfatal MI, cardiovascular and total mortality in high risk patients, while ARB modestly reduced stroke with no effect on nonfatal MI, cardiovascular and total mortality.
\end{abstract}

\section{Introduction}

Angiotensin-converting enzyme inhibitors (ACEIs) and angiotensin-receptor blockers (ARBs) have been shown to reduce cardiovascular outcomes in patients with heart failure or hypertension [1-3]. However, whether ACEI and ARB are useful in reducing cardiovascular events amongst patients at risk from a variety of clinical conditions but without left ventricular systolic dysfunction is more debatable. Several meta-analyses have addressed this issue, but these solely reviewed either ACEI or ARB alone or looked at patients with a single disease condition like hypertension or ischemic heart disease [4-10]. Both ACEI and ARB produce inhibition of the rennin-angiotensin system and have been shown to be equivalent in their blood pressure lowering effect [11].
We thus seek to answer the question of whether ACEI and $\mathrm{ARB}$ are useful and equivalent in their reduction of total mortality, cardiovascular mortality, nonfatal myocardial infarction (MI), and stroke in patients with normal systolic function and who are at high risk of cardiovascular events from a combination of various clinical conditions.

\section{Methods}

This present meta-analysis seeks to address the question of whether ACEI and ARB should be routinely used in patients at high risk of adverse cardiovascular events; the Heart Outcomes Prevention Evaluation Study (HOPE) is the pioneering trial addressing this subject [12]. High-risk 
patients are those with a combination of cardiovascular risk factors such as hypertension, diabetes, dyslipidemia, or presence of prior clinical atheromatous condition such as coronary, cerebrovascular, or peripheral arterial disease. We excluded trials in which patients were recruited based on the presence of a specific disease condition-hypertension, heart failure, diabetes, or acute $\mathrm{MI}$ - as the usefulness of ACEI or ARB in a single condition is not the subject we are presently investigating; the antihypertensive and lipidlowering treatment to prevent heart attack trial (ALLHAT) which is a study of hypertension exemplifies the type of trials that we wish to exclude [13]. We only included trials where the end-points studied were clinical cardiovascular outcomes. We omitted ONTARGET and similar trials which compared ACEI treatment with ARB and combination therapy. These are not placebo-controlled studies similar to our other trials analysed but actually compared different treatment strategies with each other.

A PubMed search was conducted for trials published from 1990, first using the search terms "Angiotensin Receptor Antagonists" OR "Angiotensin-Converting Enzyme Inhibitors" followed by using "coronary artery disease" OR "cardiovascular disease" OR "coronary angioplasty" OR "stroke" OR "transient ischemic attack" OR "TIA" OR "peripheral vascular disease" OR "high risk" followed by "cardiovascular event" OR "cardiovascular death" OR "cardiovascular mortality" OR "myocardial infarction" OR “death" OR “mortality” OR “total mortality”. A total of 573 publications were identified. We excluded observational trials, substudy reports, or studies primarily involving biomarkers or imaging modalities. We found 475 prospective randomized controlled trials with clinical end-points. We selected prospective, randomized, placebo-controlled clinical trials recruiting high-risk patients involving at least 1200 patients followed-up for at least 2 years and excluded trials studying patients with a single specific condition or risk factor such as hypertension, diabetes, or heart failure as the usefulness of ACEI or ARB in each of these specific conditions is not the subject we presently seek to investigate (Figure 1).

Ten trials fulfilled our inclusion and exclusion criteria. The trials were assessed for risk of bias based on the presence or absence allocation concealment, blinding of the participant, care-giver, researcher, and outcome assessor, loss to follow up of $<5 \%$, and use of intention to treat analysis. Outcomes were independently extracted from the trials using a specially designed data extraction form. All authors were involved in data analysis and write up.

Meta-analysis was performed using the RevMan 5 program [14]. Mantel-Haenszel analysis was done using a fixed effects model. We analyzed the effect of treatment on allcause mortality, cardiovascular mortality, nonfatal MI, and stroke. Results were expressed as relative risks (RR) and 95\% confidence intervals (CI). Three RRs were calculated for each outcome, one for all trials and one each for trials involving ACEI and trials involving ARB. We used the $I$-squared statistical test to explore for heterogeneity. Where heterogeneity was found we attempted to explain this clinically. We intended to use a random effects model if $I$-square was greater than $60 \%$.

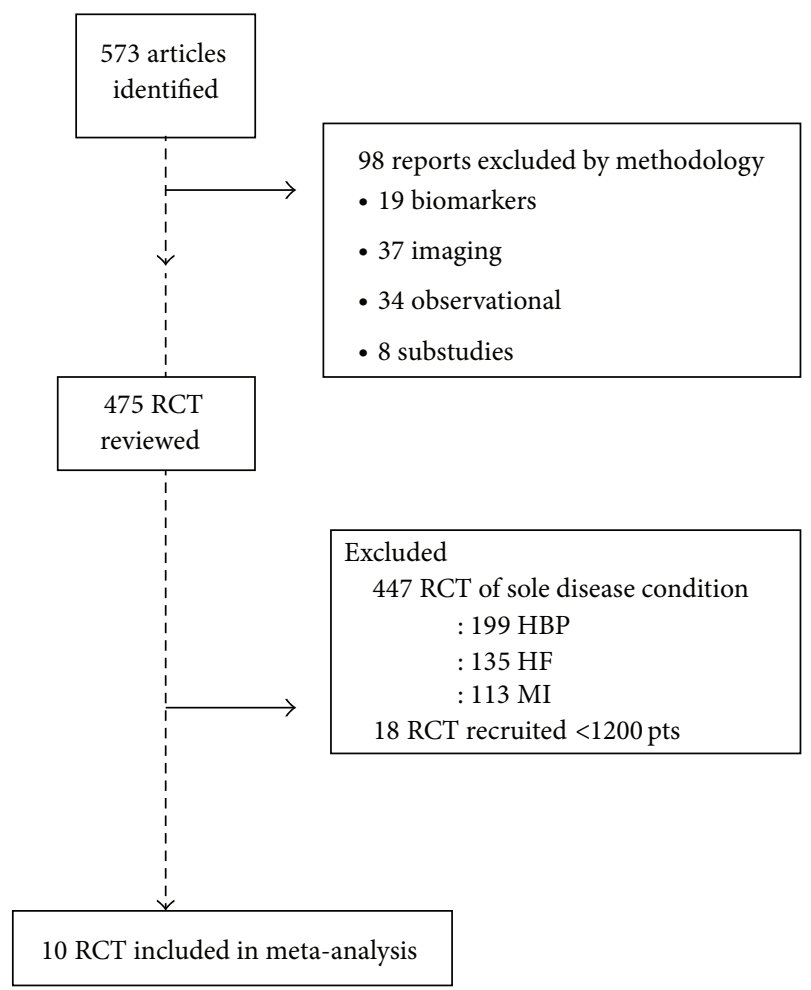

FIGURE 1: Search strategy. RCT: randomized controlled trials; HBP: hypertension; HF: heart failure; MI: myocardial infarction; pts: patients.

\section{Results}

Ten trials-HOPE, PROGRESS, QUIET, EUROPA, CAMELOT, PEACE, JIKEI, TRANSEND, PROFESS and NAVIGATOR-were included in this meta-analysis [12, 1523]. All trials were prospective, randomized, placebocontrolled trials. All had adequate allocation concealment, were blinded to participant, researcher, caregiver, and outcome assessor, and all were analyzed on an intention to treat basis. Less than $1 \%$ of those enrolled were lost to followup or withdrew after randomization in all trials reviewed except in PEACE, where $1.6 \%$ of patients were lost to followup.

The baseline characteristics of patients in the trials are as shown in Table 1 . A total of 77,633 patients were enrolled in the 10 trials, with mean followup ranging from 2 to 5 years. Half of the recruited patients were in trails comparing ACEI with placebo (38988 patients; 50.2\%), with the remainder involved in trials comparing ARB with placebo (38645 patients; $49.8 \%$ ). The recruited patients were at high risk of cardiovascular outcomes because of prior atheromatous disease (coronary, cerebrovascular, or peripheral arterial) or else had multiple risk factors for cardiovascular ischemic events. The proportion of trial patients with diabetes varied from $0 \%$ to $38 \%$, while the proportion with hypertension ranged from $27 \%$ to $88 \%$ (Table 1 ).

3.1. All-Cause Mortality. All-cause mortality was significantly reduced in trials comparing ACEI with placebo (7.67\% versus 


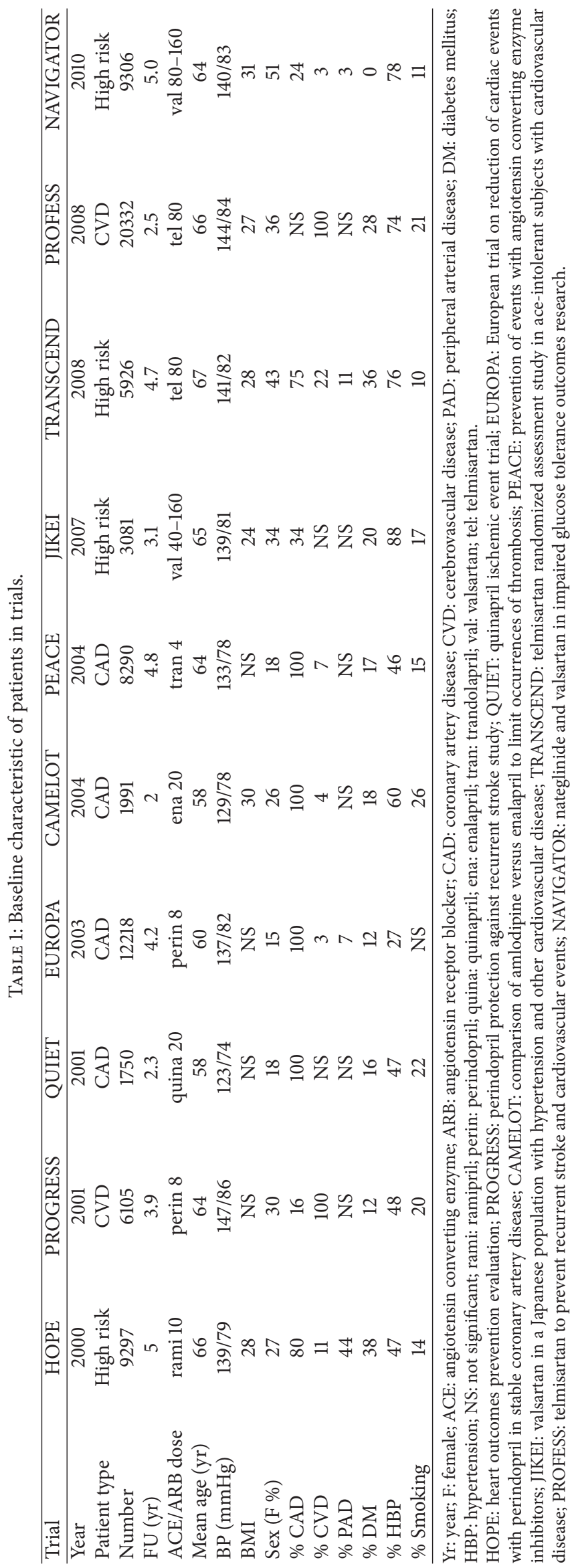


8.6\%; RR 0.89, 95\% CI 0.84-0.95; $P=0.0008$ ) but was not significantly changed in the ARB-placebo trials (7.48\% versus 7.45\%; RR 1.00, 0.94-1.08; $P=0.89$ ). No heterogeneity was noted in either the ACEI or ARB trials included in this metaanalysis. Thus, ACEI but not ARB appears to reduce total mortality in high-risk patients (Figure 2(a)).

3.2. Cardiovascular Mortality. Cardiovascular mortality was significantly reduced in the ACEI-placebo trials (4.31\% versus 5.09\%; RR 0.85, 0.78-0.93; $P=0.0003$ ) but was not significantly affected by ARB treatment (3.05\% versus $3.15 \%$; RR 0.97, 0.86-1.08; $P=0.54$ ). There was no heterogeneity in each group of trials analyzed. In patients at high risk, ACEI but not ARB significantly reduced cardiovascular mortality (Figure 2(b)).

3.3. Nonfatal MI. Compared to placebo, ACEI treatment significantly reduced nonfatal $\mathrm{MI}$ in patients at high risk (5.55\% versus $6.79 \%$; $\mathrm{RR} 0.82,0.76-0.88 ; P<0.00001)$. ARB therapy did not affect incidence of nonfatal MI (2.28\% versus $2.45 \%$; RR $0.93,0.82-1.06 ; P=0.26)$. No heterogeneity was noted within the ACEI and ARB trials. In patients at high risk, ACEI but not ARB significantly reduced nonfatal MI (Figure 2(c)).

3.4. Stroke. Stroke was significantly reduced in the ACEIplacebo trials (3.43\% versus $4.58 \%$; RR $0.75,0.68-0.83$; $P<$ $0.00001)$ and to a lesser but still significant degree in the ARB-placebo trials (5.84\% versus 6.45\%; RR 0.90, 0.84-98; $P=0.01)$. No heterogenicity was noted within ACEI trials but there was modest heterogeneity in the ARB trials. This is because the definition of cerebrovascular event in JIKEI included transient ischemic attacks, unlike in the other trials [20]. This heterogeneity disappeared when the JEKEI study was excluded, although there was no substantial change in the RR ( 0.90 with and 0.92 without JEKEI). Thus, both ACEI and $A R B$ reduce stroke incidence, although the effect from ACEI is greater (Figure $2(\mathrm{~d})$ ).

\section{Discussion}

It is important to appreciate that, despite overlapping patient characteristics, the trials selected are different from the studies of hypertension or those recruiting patients all having a specific disease or risk factor. Our target patient at high risk of cardiovascular events can have a combination of clinical conditions and risk factors but not all will have a particular condition like hypertension or dyslipidemia. Studying highrisk patients as a specific group was a novel idea until the HOPE trial. There was in fact much debate that the positive results from HOPE were due to the BP lowering effect of ramipril $[24,25]$. The fact that less than $50 \%$ of patients in HOPE had hypertension argues against the benefit coming solely from hypertension control. We feel there is a need to distinguish such high-risk patients as recruited in HOPE from those recruited into hypertensive or dyslipidemic or diabetic trials, which are designed to gather information about management of a specific disease condition. In seeking to answer the question of whether ACEI or ARB therapy is able to reduce adverse cardiovascular outcomes in patients at high risk, it is important that we analyse only the prospective, randomised, placebo-controlled trials that actually address this issue. Thus, we excluded ONTARGET and similar trials that had no placebo arm but compared active ACEI therapy with $\mathrm{ARB}$ or their combination. These trials are a comparison of different strategies of rennin-antagonism and do not answer the question we are addressing.

Our meta-analysis has shown that ACEI and ARB are not equivalent in their effect on clinical outcomes. In high-risk patients, compared to placebo, ACEI treatment significantly reduced total mortality, cardiovascular mortality, nonfatal MI, and stroke. Our meta-analysis also shows that in highrisk patients, when compared to placebo, ARB treatment has no significant effect on cardiovascular or total mortality, as well as nonfatal MI. Calculation of the needed to treat (NNT) allows a comparison of the clinical impact of ACEI with $\mathrm{ARB}$ in stroke reduction. The small benefit from ARB $(5.84 \%$ versus $6.45 \%$; NNT 164) in reducing stroke is less pronounced than the effect obtained from ACEI therapy (3.43\% versus $4.58 \%$; NNT 87). It thus appears that the ARB is inferior to the ACEI and cannot be considered its therapeutic alternative when contemplating reduction of adverse cardiovascular clinical outcomes in the high-risk group. This contrasts with the situation in heart failure where the ARB is interchangeable with ACEI [2]. Thus, in patients at high risk of coronary events, ACEI should be offered before ARB, which should be used only in those intolerant of ACEI.

However, the benefit of ACEI in reducing clinical cardiovascular events in the high-risk group is modest. The NNT to prevent total death is 108 , to prevent cardiovascular death is 128 , to prevent a nonfatal MI is 81 , and to prevent stroke is 87 . The NNT to prevent total mortality is 42 with betablockers after MI and is between 25-56 with simvastatin in the secondary prevention trials [26-28]. ACEI are more effective in preventing mortality in patients with impaired LV function; the NNT for total mortality is 22 using enalapril in patients with heart failure and an EF below 35\% [1]. Thus, while ACEI are first line drugs in patients with heart failure and poor LV function, our data suggest they should be used only after statins and beta-blockers when seeking to reduce mortality and adverse clinical events in those with normal systolic function at high cardiovascular risk.

It is important in pooling studies that the statistical methodology employed does not lead to incorrect results, and authors do not produce overall conclusions that are inconsistent with the individual data $[25,26]$. In our metaanalysis, the trials pooled together did not exhibit any heterogeneity, allowing greater confidence in pooling them together and in the validity of the overall findings. ACEI and ARB have been compared to other agents in patients with poor systolic function, hypertension, and diabetic nephropathy [2, 27-37]. We intentionally omitted such trials from our analysis as these patients are different from the group we have addressed, which are patients with high risk for or with past history of clinical atheromatous disease. The $95 \%$ confidence interval (CI) for most of the trials analyzed is narrow. In the ACEI-placebo trials, the midpoint of the CI 


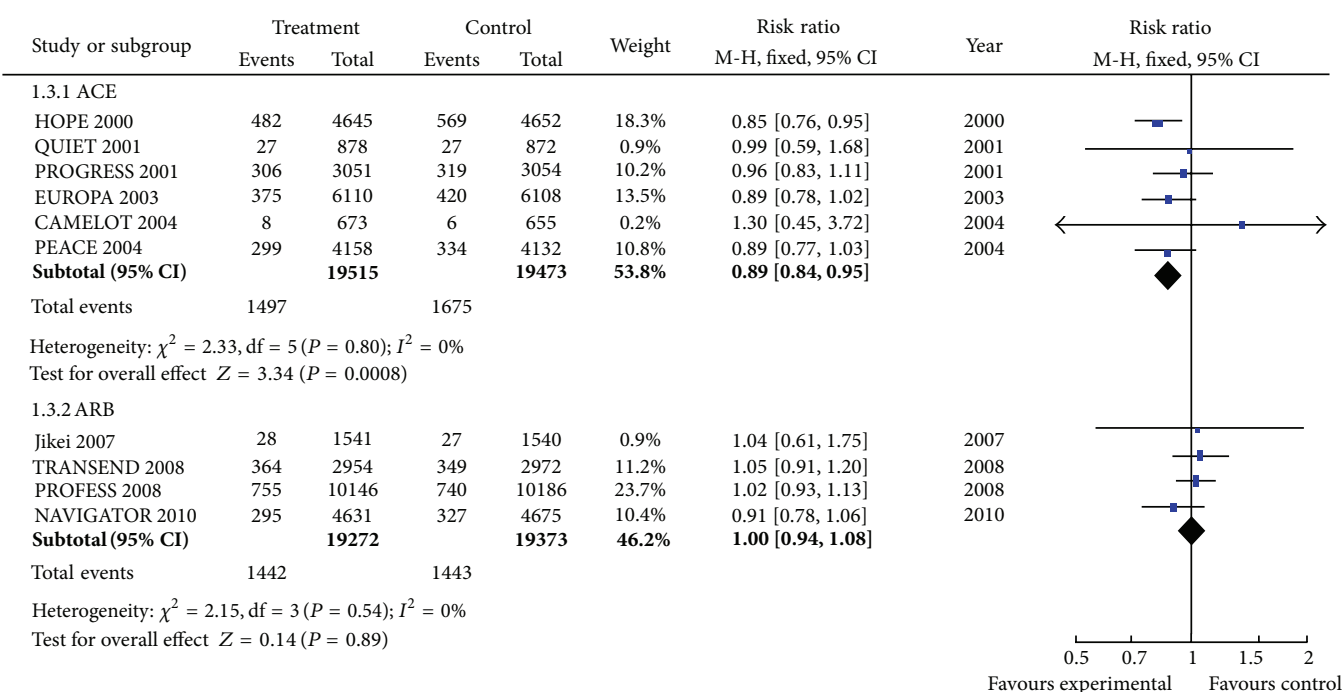

(a)

\begin{tabular}{lccccccr} 
& \multicolumn{2}{c}{ Treatment } & \multicolumn{2}{c}{ Control } & \multirow{2}{*}{ Risk ratio } & Year \\
Study or subgroup & Events & Total & Events & Total & Weight & M-H, fixed, 95\% CI & \\
& & & & & & & \\
1.2.1 ACE & 282 & 4645 & 377 & 4652 & $23.5 \%$ & $0.75[0.65,0.87]$ & 2000 \\
HOPE 2000 & 181 & 3051 & 198 & 3054 & $12.4 \%$ & $0.92[0.75,1.11]$ & 200 \\
PROGRESS 2001 & 13 & 878 & 14 & 872 & $0.9 \%$ & $0.92[0.44,1.95]$ & 200 \\
QUIET 2001 & 215 & 6110 & 249 & 6108 & $15.6 \%$ & $0.86[0.72,1.03]$ & 2003 \\
EUROPA 2003 & 146 & 4158 & 152 & 4132 & $9.5 \%$ & $0.95[0.76,1.19]$ & 2004 \\
PEACE 2004 & 5 & 673 & 2 & 655 & $0.1 \%$ & $2.43[0.47,12.50]$ & 2004 \\
CAMELOT 2004 & & $\mathbf{1 9 5 1 5}$ & & $\mathbf{1 9 4 7 3}$ & $\mathbf{6 2 . 0 \%}$ & $\mathbf{0 . 8 5}[\mathbf{0 . 7 8 , 0 . 9 3}]$ & \\
Subtotal (95\% CI) & & & 992 & & & &
\end{tabular}

Heterogeneity: $\chi^{2}=6.01, \mathrm{df}=5(P=0.31) ; I^{2}=17 \%$

Test for overall effect $Z=3.61(P=0.0003)$

1.2.2ARB

Jikei 2007

PROFESS 2008

$\begin{array}{ccc}9 & 1541 & 9 \\ 223 & 10146 & 263 \\ 227 & 2954 & 223 \\ 128 & 4631 & 11\end{array}$

$9 \quad 1540 \quad 0.6 \%$

$\begin{array}{cc}10186 & 16.4 \% \\ 2972 & 13.9 \%\end{array}$

$13.9 \%$

NAVIGATOR 2010

4631

$4675 \quad 7.2 \%$

$0.85[0.71,1.02]$

$1.02[0.86,1.22]$

$1.11[0.87,1.43]$

Subtotal $(95 \% \mathrm{CI})$

587 611

$0.97[0.86,1.08]$

Heterogeneity: $\chi^{2}=3.67, \mathrm{df}=3(P=0.30) ; I^{2}=18 \%$

Test for overall effect $Z=0.61(P=0.54)$

(9373

Risk ratio

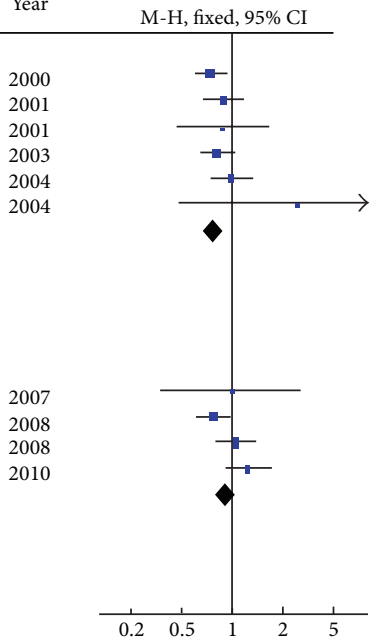

Favours experimental Favours control

(b)

\begin{tabular}{|c|c|c|c|c|c|c|c|c|}
\hline \multirow{2}{*}{ Study or subgroup } & \multicolumn{2}{|c|}{ Treatment } & \multicolumn{2}{|c|}{ Control } & \multirow{2}{*}{ Weight } & \multicolumn{2}{|l|}{ Risk ratio } & \multirow{2}{*}{$\begin{array}{c}\text { Risk ratio } \\
\text { M-H, fixed, } 95 \% \mathrm{CI}\end{array}$} \\
\hline & Events & Total & Events & Total & & M-H, fixed, 95\% CI & Year & \\
\hline \multicolumn{9}{|l|}{ 1.4.1 ACE } \\
\hline HOPE 2000 & 459 & 4645 & 570 & 4652 & $31.7 \%$ & $0.81[0.72,0.91]$ & 2000 & $\rightarrow$ \\
\hline PROGRESS 2001 & 60 & 3051 & 96 & 3054 & $5.3 \%$ & $0.63[0.45,0.86]$ & 2001 & 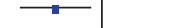 \\
\hline QUIET 2001 & 36 & 878 & 40 & 872 & $2.2 \%$ & $0.89[0.58,1.39]$ & 2001 & \\
\hline EUROPA 2003 & 295 & 6110 & 378 & 6108 & $21.0 \%$ & $0.78[0.67,0.90]$ & 2003 & $\rightarrow-$ \\
\hline PEACE 2004 & 222 & 4158 & 220 & 4132 & $12.3 \%$ & $1.00[0.84,1.20]$ & 2004 & ] \\
\hline CAMELOT 2004 & 11 & 673 & 19 & 655 & $1.1 \%$ & $0.56[0.27,1.17]$ & 2004 & \\
\hline Subtotal (95\% CI) & & 19515 & & 19473 & $73.7 \%$ & $0.82[0.76,0.88]$ & & 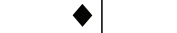 \\
\hline Total events & 1083 & & 1323 & & & & & \\
\hline \multicolumn{9}{|c|}{ Heterogeneity: $\chi^{2}=9.16, \mathrm{df}=5(P=0.10) ; I^{2}=45 \%$} \\
\hline \multicolumn{9}{|c|}{ Test for overall effect $Z=5.11(P<0.00001)$} \\
\hline \multicolumn{9}{|l|}{ 1.4.2ARB } \\
\hline Jikei 2007 & 17 & 1541 & 19 & 1540 & $1.1 \%$ & $0.89[0.47,1.71]$ & 2007 & 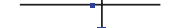 \\
\hline PROFESS 2008 & 168 & 10146 & 169 & 10186 & $9.4 \%$ & $1.00[0.81,1.23]$ & 2008 & \\
\hline TRANSEND 2008 & 116 & 2954 & 147 & 2972 & $8.2 \%$ & $0.79[0.63,1.01]$ & 2008 & 1 \\
\hline NAVIGATOR 2010 & 138 & 4631 & 140 & 4675 & $7.8 \%$ & $1.00[0.79,1.25]$ & 2010 & $\rightarrow$ \\
\hline Subtotal $(95 \% \mathrm{CI})$ & & 19272 & & 19373 & $26.3 \%$ & $0.93[0.82,1.06]$ & & 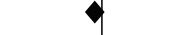 \\
\hline Total events & 439 & & 475 & & & & & \\
\hline \multicolumn{9}{|c|}{ Heterogeneity: $\chi^{2}=2.47, \mathrm{df}=3(P=0.48) ; I^{2}=0 \%$} \\
\hline Test for overall effect & $=1.11(P$ & $=0.26)$ & & & & & & \\
\hline
\end{tabular}

(c)

Figure 2: Continued. 


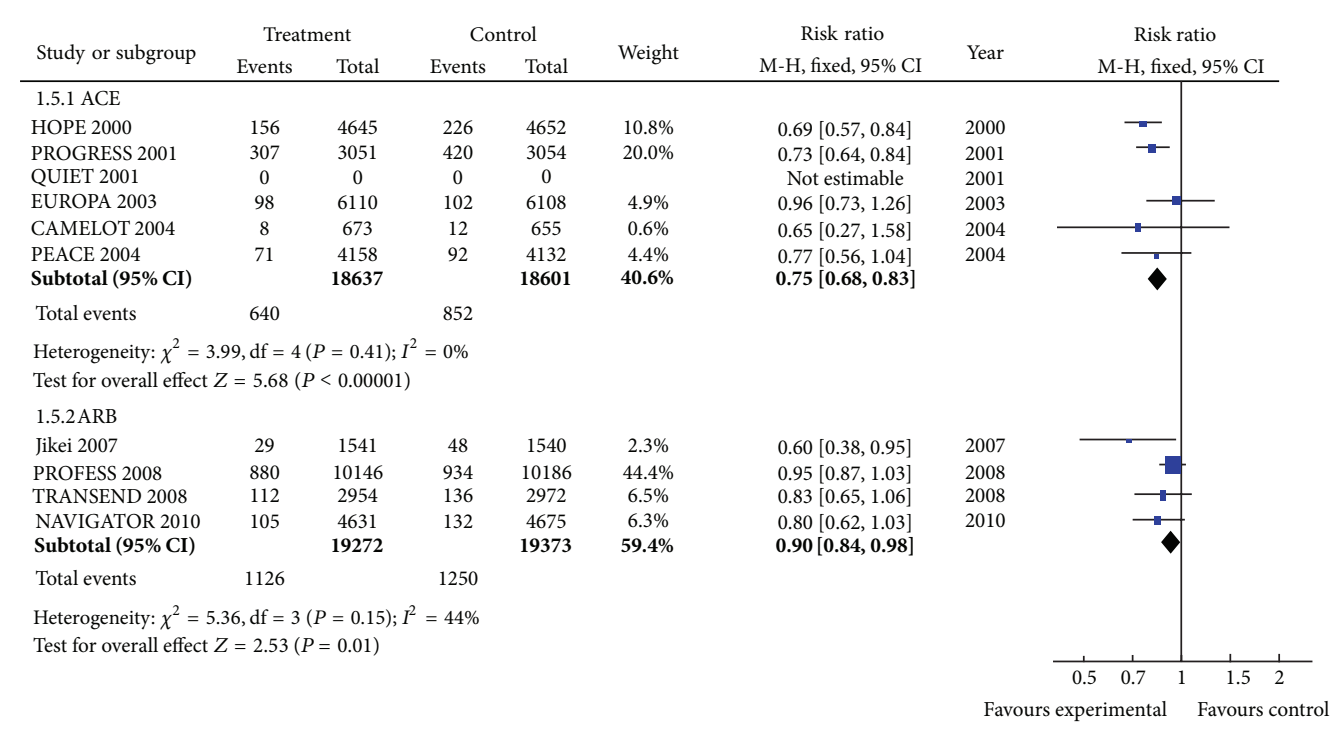

(d)

FIgure 2: (a) Total mortality. (b) Cardiovascular mortality. (c) Nonfatal myocardial infarction. (d) Total stroke. HOPE: heart outcomes prevention evaluation; PROGRESS: perindopril protection against recurrent stroke study; QUIET: quinapril ischemic event trial; EUROPA: European trial on reduction of cardiac events with perindopril in stable coronary artery disease; CAMELOT: comparison of amlodipine versus enalapril to limit occurrences of thrombosis; PEACE: prevention of events with angiotensin converting enzyme inhibitors; JIKEI: valsartan in a Japanese population with hypertension and other cardiovascular disease; TRANSCEND: telmisartan randomized assessment study in ACE-intolerant subjects with cardiovascular disease; PROFESS: telmisartan to prevent recurrent stroke and cardiovascular events; NAVIGATOR: nateglinide and valsartan in impaired glucose tolerance outcomes research. HOPE [12] PROGRESS [15] QUIET [16] EUROPA [17] CAMELOT [18] PEACE [19] JIKEI [20] TRANSCEND [21] PROFESS [22] NAVIGATOR [23].

in most of the individual studies point to a benefit from ACEI therapy agreeing with the pooled conclusion; in the ARB trials, the individual midpoints are very close to 1 and thus are in agreement at the absence of any treatment effect. The confidence in the correctness of our conclusion is thus enhanced. In patients at high risk of clinical atheromatous disease, these two groups of drugs do not have the same effect. ACEI reduced stroke, nonfatal MI, and cardiovascular and total mortality in high-risk patients, while ARB only modestly reduced stroke and has no effect on nonfatal MI, cardiovascular, and total mortality.

\section{Conflict of Interests}

The authors have no competing, conflicting, or funding interests to report.

\section{References}

[1] The SOLVD Investigators, "Effect of enalapril on survival in patients with reduced left ventricular ejection fractions and congestive heart failure," The New England Journal of Medicine, vol. 325, no. 5, pp. 293-302, 1991.

[2] C. B. Granger, J. J. V. McMurray, S. Yusuf et al., "Effects of candesartan in patients with chronic heart failure and reduced left-ventricular systolic function intolerant to angiotensinconverting-enzyme inhibitors: the CHARM-alternative trial," The Lancet, vol. 362, no. 9386, pp. 772-776, 2003.
[3] Blood Pressure Lowering Treatment Trialists' Collaboration, "Effects of different blood-pressure-lowering regimens on major cardiovascular events: results of prospectively-designed overviews of randomised trials," The Lancet, vol. 362, no. 9395, pp. 1527-1535, 2003.

[4] M. H. Al-Mallah, I. M. Tleyjeh, A. A. Abdel-Latif, and W. D. Weaver, "Angiotensin-converting enzyme inhibitors in coronary artery disease and preserved left ventricular systolic function. A systematic review and meta-analysis of randomized controlled trials," Journal of the American College of Cardiology, vol. 47, no. 8, pp. 1576-1583, 2006.

[5] G. R. Dagenais, J. Pogue, K. Fox, M. L. Simoons, and S. Yusuf, "Angiotensin-converting-enzyme inhibitors in stable vascular disease without left ventricular systolic dysfunction or heart failure: a combined analysis of three trials," The Lancet, vol. 368, no. 9535, pp. 581-588, 2006.

[6] N. Danchin, M. Cucherat, C. Thuillez, E. Durand, Z. Kadri, and P. G. Steg, "Angiotensin-converting enzyme inhibitors in patients with coronary artery disease and absence of heart failure or left ventricular systolic dysfunction: an overview of long-term randomized controlled trials," Archives of Internal Medicine, vol. 166, no. 7, pp. 787-796, 2006.

[7] M. Volpe, G. Tocci, S. Sciarretta, P. Verdecchia, B. Trimarco, and G. Mancia, "Angiotensin II receptor blockers and myocardial infarction: an updated analysis of randomized clinical trials," Journal of Hypertension, vol. 27, no. 5, pp. 941-946, 2009.

[8] M. M. Al Khalaf, L. Thalib, and S. A. R. Doi, "Cardiovascular outcomes in high-risk patients without heart failure treated with ARBs: a systematic review and meta-analysis," American Journal of Cardiovascular Drugs, vol. 9, no. 1, pp. 29-43, 2009. 
[9] L. C. van Vark, M. Bertrand, K. M. Akkerhius et al., "Angiotensin-converting enzyme inhibitors reduce mortality in hypertension: a meta-analysis of randomized clinical trials of renninangiotensin-aldosterone system inhibitors involving 158,998 patients," European Heart Journal, vol. 33, pp. 2088-2097, 2012.

[10] W. L. Baker, C. I. Coleman, J. Kluger et al., "Systematic review: comparative effectiveness of angiotensin-converting enzyme inhibitors or angiotensin II-receptor blockers for ischemic heart disease," Annals of Internal Medicine, vol. 151, no. 12, pp. 861-871, 2009.

[11] The ONTARGET Investigators, "Telmisartan, ramipril or both in patients at high risk for vascular events," The New England Journal of Medicine, vol. 358, pp. 1547-1559, 2008.

[12] The Heart Outcomes Prevention Evaluation Study Investigators, "Effects of an angiotensin-converting-enzyme inhibitor, ramipril, on cardiovascular events in high-risk patients," The New England Journal of Medicine, vol. 342, no. 3, pp. 145-153, 2000.

[13] ALLHAT Officers and Coordinators for the ALLHAT Collaborative Research Group, "Major outcomes in high-risk hypertensive patients randomized to angiotensin-converting enzyme inhibitor or calcium channel blocker vs diuretic: the Antihypertensive and Lipid-Lowering Treatment to Prevent Heart Attack Trial (ALLHAT)," JAMA, vol. 288, pp. 2981-2997, 2002.

[14] Review Manager (RevMan) [Computer program], Version 5.0. Copenhagen: The Nordic Cochrane Centre, The Cochrane Collaboration, 2008.

[15] PROGRESS Collaborative Group, "Randomised trial of a perindopril-based blood pressure lowering regimen among 6105 individuals with previous stroke or transient ischemic attack," The Lancet, vol. 358, pp. 1033-1041, 2001.

[16] B. Pitt, B. O’Neill, R. Feldman et al., “The Quinapril Ischemic Event Trial (QUIET): evaluation of chronic ACE inhibitor therapy in patients with ischemic heart disease and preserved left ventricular function," American Journal of Cardiology, vol. 87, no. 9, pp. 1058-1063, 2001.

[17] EURopean Trial on Reduction of Cardiac Events with Perindopril in Stable Coronary Artery Disease Investigators, "Efficacy of perindopril in reduction of cardiovascular events among patients with stable coronary artery disease: randomised, double-blind, placebo-controlled, multicentre trial (the EUROPA study)," The Lancet, vol. 362, no. 9386, pp. 782$788,2003$.

[18] S. E. Nissen, E. M. Tuzcu, P. Libby et al., "Effect of antihypertensive agents on cardiovascular events in patients with coronary disease and normal blood pressure. The CAMELOT study: a randomized controlled trial," Journal of the American Medical Association, vol. 292, no. 18, pp. 2217-2226, 2004.

[19] The PEACE Trial Investigators, "Angiotensin-converting enzyme inhibition in stable Coronary Artery Disease," The New England Journal of Medicine, vol. 351, pp. 2058-2068, 2004.

[20] S. Mochizuki, B. Dahlöf, M. Shimizu et al., "Valsartan in a Japanese population with hypertension and other cardiovascular disease (Jikei Heart Study): a randomised, open-label, blinded endpoint morbidity-mortality study," The Lancet, vol. 369, no. 9571, pp. 1431-1439, 2007.

[21] The Telmisartan Randomised Assessment Study in ACE Intolerant Subjects with Cardiovascular Disease (TRANSCEND) Investigators, "Effects of the angiotensin-receptor blocker telmisartan on cardiovascular events in high-risk patients intolerant to angiotensin-converting enzyme inhibitors: a randomized controlled trial," The Lancet, vol. 372, pp. 1174-1183, 2008.

[22] S. Yusuf, H. C. Diener, R. L. Sacco et al., “Telmisartan to prevent recurrent stroke and cardiovascular events," The New England Journal of Medicine, vol. 359, pp. 1225-1237, 2008.

[23] The NAVIGATOR Study Group, "Effect of valsartan on the incidence of diabetes and cardiovascular events," The New England Journal of Medicine, vol. 362, pp. 1463-1476, 2010.

[24] P. Svensson, U. de Faire, P. Sleight, S. Yusuf, and J. Ostergren, "Comparative effects of ramipril on ambulatory and office blood pressures: a HOPE Substudy, Hypertension, vol. 38, no. 6, pp. E28-E32, 2001.

[25] G. D. Moutsatsos, "More hype than HOPE," Hypertension, vol. 41, no. 4, pp. e4-e5, 2003.

[26] N. Freemantle, J. Cleland, P. Young, J. Mason, and J. Harrison, " $\beta$ blockade after myocardial infarction: systematic review and meta regression analysis," British Medical Journal, vol. 318, no. 7200, pp. 1730-1737, 1999.

[27] Scandinavian Simvastatin Survival Study Group, "Randomised trial of cholesterol lowering in 4444 patients with coronary heart disease: the Scandinavian Simvastatin Survival Study (4S)," The Lancet, vol. 344, no. 8934, pp. 1383-1389, 1994.

[28] Heart Protection Study Collaborative Group, "MRC/BHF Heart Protection Study of cholesterol lowering with simvastatin in 5963 people with diabetes: a randomized placebo controlled trial," The Lancet, vol. 361, pp. 2005-2016, 2003.

[29] A. N. DeMaria, "Lies, damned lies, and statistics," Journal of the American College of Cardiology, vol. 52, no. 17, pp. 1430-1431, 2008.

[30] I. Boutron, S. Dutton, P. Ravaud, and D. G. Altman, "Reporting and interpretation of randomized controlled trials with statistically nonsignificant results for primary outcomes," Journal of the American Medical Association, vol. 303, no. 20, pp. 2058-2064, 2010.

[31] J. N. Cohn and G. Tognoni, "A randomized trial of the angiotensin-receptor blocker valsartan in chronic heart failure," The New England Journal of Medicine, vol. 345, no. 23, pp. 16671675, 2001.

[32] UK Prospective Diabetes Study Group, "Efficacy of atenolol and captopril in reducing risk of macrovascular and microvascular complications in type 2 diabetes: UKPDS 39," British Medical Journal, vol. 317, no. 7160, pp. 713-720, 1998.

[33] L. Hansson, L. H. Lindholm, L. Niskanen et al., "Effect of angiotensin-converting-enzyme inhibition compared with conventional therapy on cardiovascular morbidity and mortality in hypertension: the Captopril Prevention Project (CAPPP) randomised trial," The Lancet, vol. 353, no. 9153, pp. 611-616, 1999.

[34] L. Hansson, L. H. Lindholm, T. Ekbom et al., "Randomised trial of old and new antihypertensive drugs in elderly patients: cardiovascular mortality and morbidity the Swedish trial in old patients with hypertension-2 study," The Lancet, vol. 354, no. 9192, pp. 1751-1756, 1999.

[35] B. Dahlöf, R. B. Devereux, S. E. Kjeldsen et al., "Cardiovascular morbidity and mortality in the Losartan Intervention For Endpoint reduction in hypertension study (LIFE): a randomised trial against atenolol," The Lancet, vol. 359, no. 9311, pp. 995$1003,2002$. 
[36] S. Julius, S. E. Kjeldsen, M. Weber et al., "Outcomes in hypertensive patients at high cardiovascular risk treated with regimens based on valsartan or amlodipine: the VALUE randomised trial," The Lancet, vol. 363, no. 9426, pp. 2022-2031, 2004.

[37] Y. Yui, T. Sumiyoshi, K. Kodama et al., "Comparison of nifedipine retard with angiotensin converting enzyme inhibitors in Japanese hypertensive patients with coronary artery disease: the Japan Multicenter Investigation for Cardiovascular Diseases-B (JMIC-B) randomized trial," Hypertension Research, vol. 27, no. 3, pp. 181-191, 2004. 


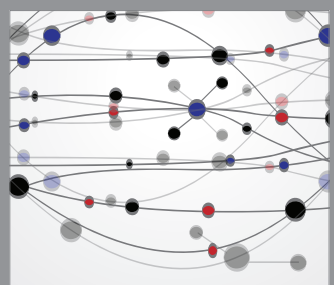

The Scientific World Journal
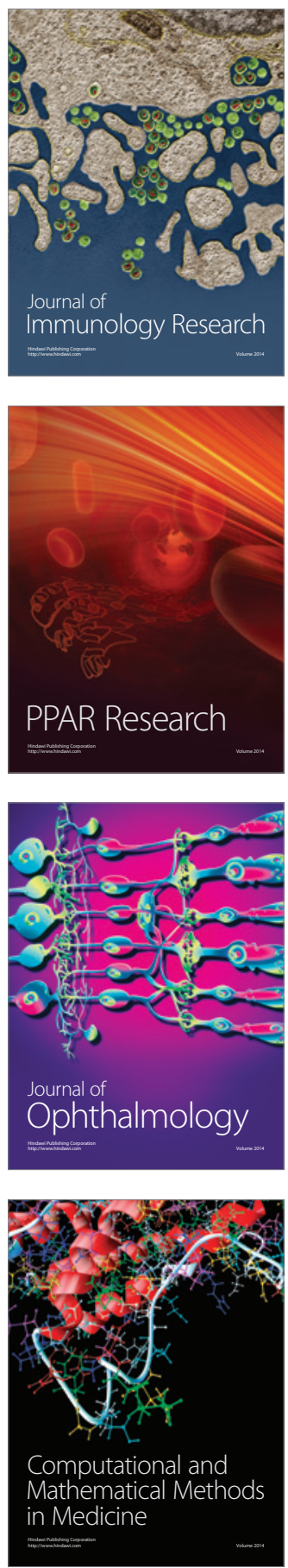

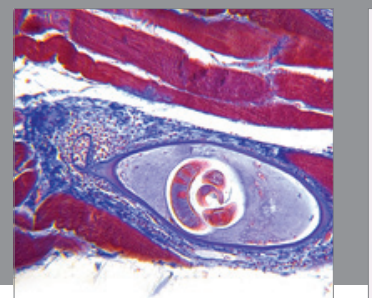

Gastroenterology

Research and Practice
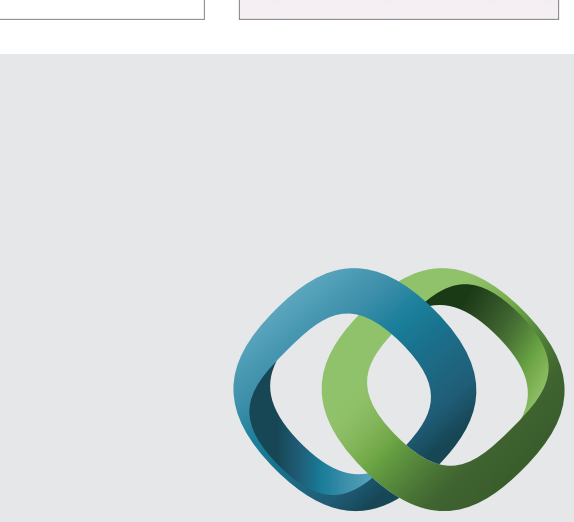

\section{Hindawi}

Submit your manuscripts at

http://www.hindawi.com
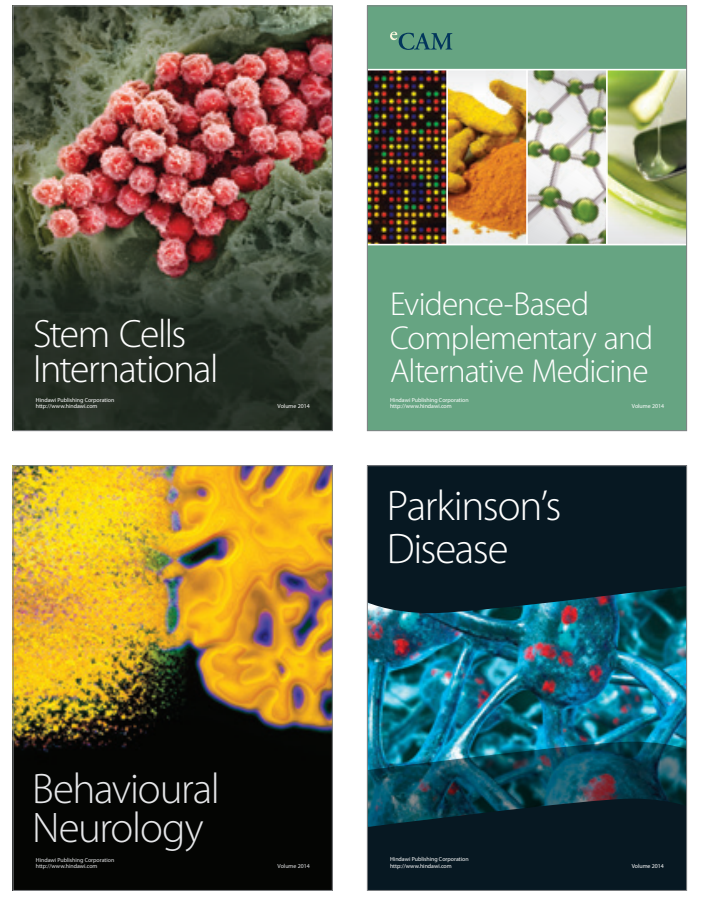
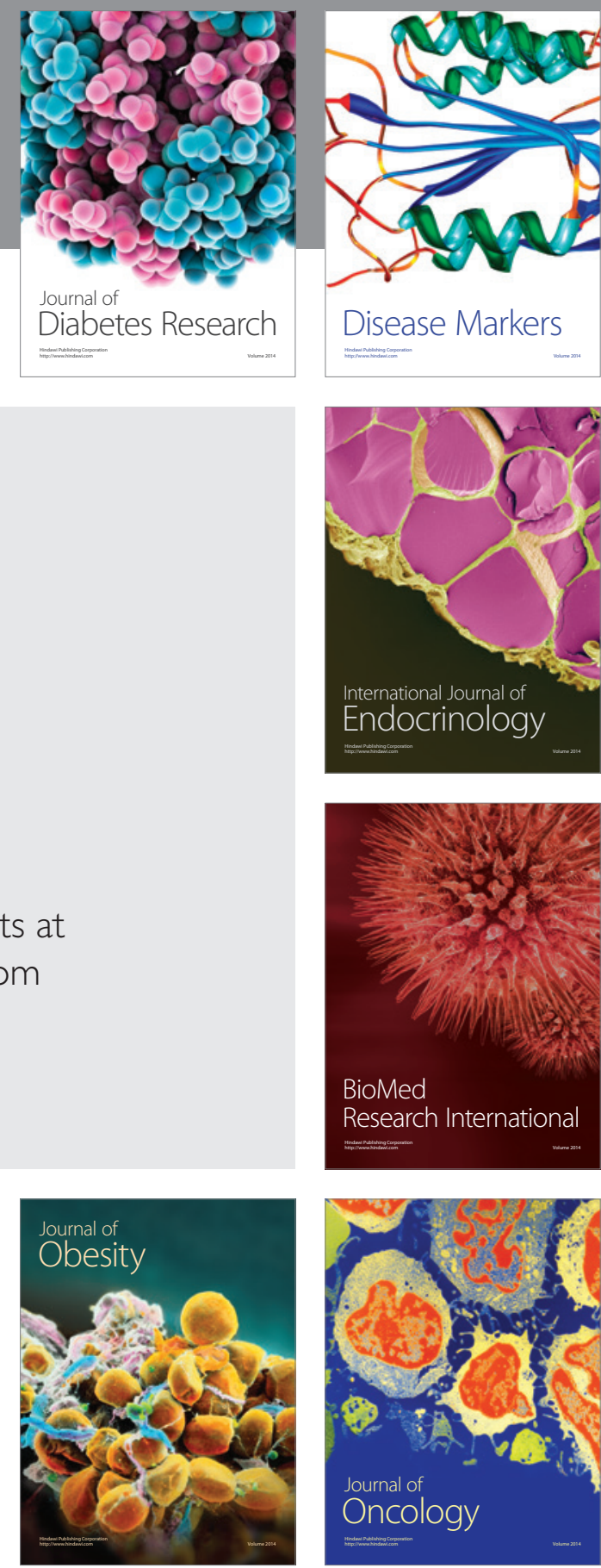

Disease Markers
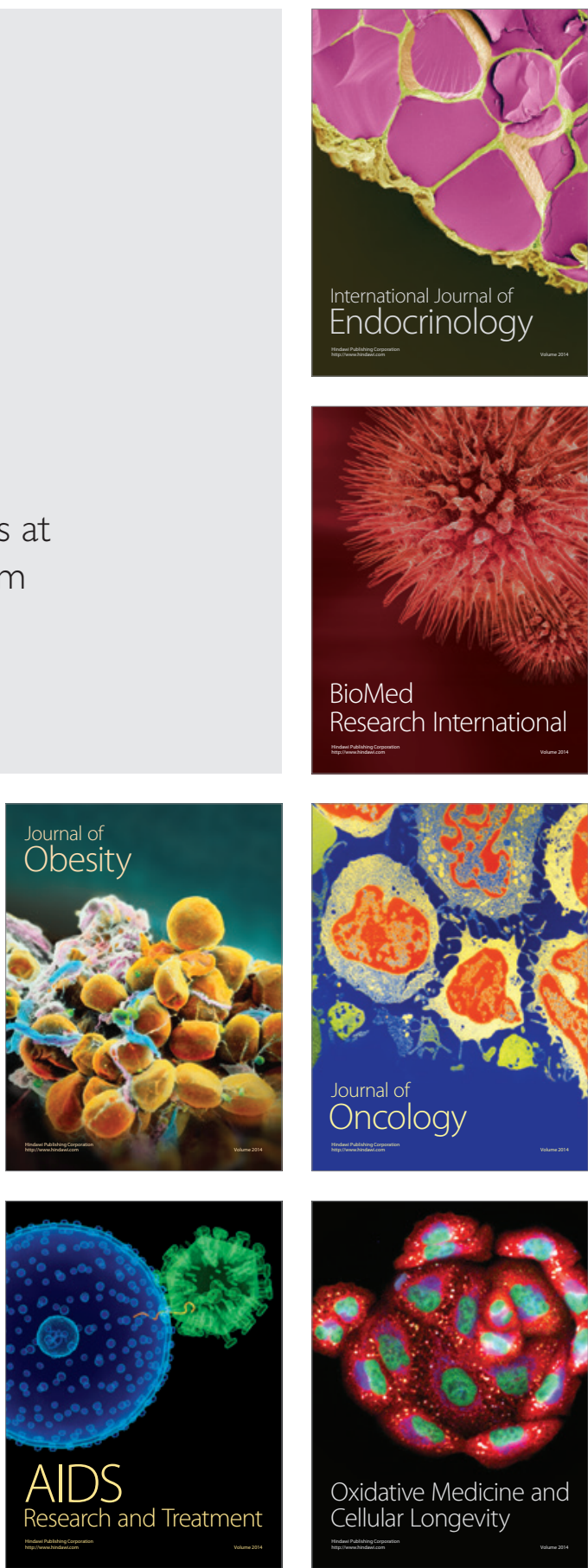